\title{
A Handheld Computer Smoking Intervention Tool and Its Effects on Physician Smoking Cessation Counseling
}

Scott M. Strayer, MD, MPH, Lisa K. Rollins, PhD, and James R. Martindale, PhD

Objective: The objective of this study was to evaluate a handheld computer smoking cessation intervention tool designed to assist physicians in their smoking cessation counseling with patients.

Methods: This study used a pre/post survey design, with a 4-month trial period for the software. Study participants included 22 faculty and resident physicians from the University of Virginia. Paired samples $t$ tests were used to assess mean differences in the 4 main subscales (physician behavior, attitudes, comfort related to counseling patients about smoking cessation, and knowledge).

Results: No statistically significant mean differences were found for physician behavior (mean increase $=0.44, P=.55$ ) or physician attitude (mean increase $=0.44, P=.16)$. A statistically significant mean increase of 2.29 was observed for the physician comfort subscale $(t=3.87, d f=16, P=$ .001). Physicians indicated improved comfort in counseling patients about smoking cessation $(P=$ $.007)$ and improved comfort in using the Public Health Service Clinical Practice Guidelines $(P=.012)$.

Conclusion: Physician comfort level in counseling patients about smoking cessation can be improved through handheld computer software. When used in conjunction with other practice modifications, this tool has the potential to improve physician smoking cessation intervention practices. (J Am Board Fam Med 2006;19:350-7.)

\section{Background}

It is well established that smoking is the leading cause of preventable disease and death in the United States. ${ }^{1}$ In addition, health care professionals have the opportunity to intervene with patients who smoke, as at least $70 \%$ of smokers visit a physician annually, ${ }^{2}$ and even brief smoking cessation counseling (less than 3 minutes) by physicians is effective. ${ }^{3,4}$ Despite this opportunity for a posi-

Submitted 10 June 2005; revised 25 January 2006; accepted 1 February 2006.

From the Departments of Family Medicine (SMS, LKR), Public Health Sciences (SMS), and Medical Education Support (JRM), University of Virginia Health System, Charlottesville, VA.

Funding: SMS received funding from a National Cancer Institute Market Requisition 263-MQ-215807 and from a Health Resources and Services Administration (HRSA) Catalog of Federal Domestic Assistance no. 93.984, Academic Units in Primary Care-Family Medicine grant. LKR was also supported by the HRSA grant. JRM is funded through the University of Virginia School of Medicine.

Conflict of interest: The Handheld Computer Smoking Intervention Tool software was developed by Dr. Strayer in partnership between the University of Virginia Department of Family Medicine and the National Cancer Institute. The authors have no other competing interests to declare.

Corresponding author: Scott M. Strayer, MD, MPH, Department of Family Medicine, University of Virginia Health System, PO Box 800729, Charlottesville, VA 22908-0729 (E-mail: sstrayer@virginia.edu). tive health intervention, tobacco cessation counseling only occurs at $23 \%$ to $46 \%$ of primary care office visits, ${ }^{5,6}$ and physician smoking cessation counseling and treatment continues to fall short of national health promotion objectives. ${ }^{5,7}$

A variety of barriers are probably contributors to limiting physician smoking intervention practices. These barriers include time constraints of appointments, lack of physician confidence in counseling smokers, and lack of physician knowledge regarding smoking cessation guidelines. ${ }^{8-12}$

In addition, many physicians are unaware of the national Treating Tobacco Use and Dependence Guidelines published by the Public Health Service, ${ }^{13}$ and they may not be aware of different available pharmacotherapies (especially newer ones and second-line treatments), dosing for smoking cessation, or available valid and reliable instruments, such as the Fagerström Test for Nicotine Dependence (FTND), which can be used to improve smoking cessation interventions. ${ }^{14-16}$ By making relevant and valid medical information readily available to physicians at the point of care, physician knowledge and comfort in applying the guidelines may improve. This, in turn, may lead to improved smoking cessation counseling behavior 
(through increased self-efficacy) and ultimately, improved patient outcomes.

One means of providing medical information to physicians is to incorporate it into a guided information system that is portable, convenient, allows rapid access, and is intuitive for the user. Handheld computers provide an ideal platform for this kind of information system because they enable rapid access to large amounts of information in a portable format. Handheld computers are used widely by physicians at the point of care to look up reference information (eg, guidelines and drug dosing), clinical calculations, tracking procedures performed and billing. ${ }^{17}$ Although handheld sales among the general population have tapered recently, it is estimated that one in 2 physicians use a handheld computer and they are 5 times more likely to carry them than average citizens. ${ }^{18}$

There is emerging evidence that handheld computers may be an effective modality in improving patient outcomes. A recent study completed in a critical care unit demonstrated that handheld computer-based decision support reduced lengths of stay for patients and also reduced antibiotic prescribing. ${ }^{19}$ Office-based studies with handheld computers have shown increased adherence to asthma guidelines, ${ }^{20}$ potential reduction in adverse drug effects, ${ }^{21}$ improved antibiotic prescribing for otitis media in children, ${ }^{22}$ and reduced inappropriate use of antibiotics for upper respiratory infections. ${ }^{23}$ In addition, a recent Robert Wood Johnson Foundation initiative, the Prescription for Health Project, was undertaken to improve patient care through innovative approaches and technologies. Several of the projects reported promising pilot results in addressing tobacco use, unhealthy diets, sedentary lifestyles, and alcohol misuse using handheld computers. ${ }^{24}$

The objective of this study was to evaluate a handheld computer smoking cessation intervention tool designed to assist physicians in their smoking cessation counseling with patients. The tool provides easy access to a variety of relevant and valid smoking cessation information and can be used by physicians at the point of care.

\section{Methods}

\section{Description of the Handheld Computer Smoking Intervention Tool (HCSIT)}

The HCSIT was designed to assist clinicians with smoking cessation counseling of patients at the point of care and included the Public Health Service Guidelines on Smoking Cessation (including various options for smoking cessation interventions and smoking cessation counseling tips), smoking cessation drug prescribing information (including prescription information for the Commit lozenge and second-line pharmacotherapy), and the FTND questionnaire that scored automatically and recommended pharmacotherapy for highly dependent smokers. A sample of the main page screen shot can be seen in Figure 1. The design of the tool enabled users to skip to the elements that would be most useful to them during the patient encounter.

Sample screen shots pertaining to the FTND can be found in Figures 2 and 3. Users were guided through the FTND using a series of screen shots that addressed each of 6 questions. Once the questions were completed, the tool tabulated the responses and generated specific intervention recommendations based on the level of dependency (Figure 3). When medication therapy was strongly indicated, common prescription information was provided to assist the physician with dispensing the appropriate medication (Figure 4). In addition, the software contained embedded log files to track frequency of use and content accessed via time and date stamps.

This software was developed in partnership between the University of Virginia Department of Family Medicine and the National Cancer Institute, in accordance with the Public Health Service guidelines. ${ }^{11}$ The program can be used with both Palm OS and Microsoft Pocket PC handheld computers and can be downloaded at $\langle$ http://www. smokefree.gov/hp-hcsit.html .

\section{Study Design}

This study used a pre/post survey design. The survey was developed by the authors and was adapted from 2 surveys that were developed at other academic institutions to assess smoking cessation counseling by health care workers (see Table 1). Face and content validity of the survey were assessed with a pilot sample of 40 physicians from a separate university in Missouri. The pre/post survey included participant demographic information, including computer literacy, age, gender, and professional status (eg, resident, faculty) and 4 subscales that measured self-reported physician behavior, attitudes, comfort related to counseling patients about smoking cessation, and knowledge 


\section{HCSIT Moin}

\section{Handheld Computer Sinoking Interuention Tool}

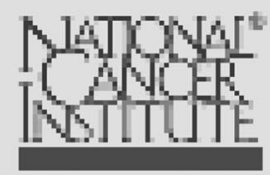

Fleose Select o file:

\section{Commit Prescribing Information} Common Smoking Cesotion Drugs Motiwational Smoking Cessotion lnt... Second-Line Pharmucotherapy Smoking Cesotion Gounseling Tip...

B

\section{HCSIT Main}

\section{Handheld Computer Smoking Interuention Tool}

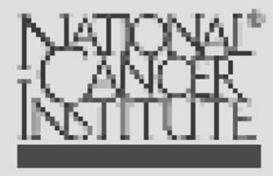

Fleose Select o file:

Wotivotional Smoking Cessotion ln...* Second-Line Phurmucotherapy Smoking Cessotion Counseling Tips -... Smoking Dependency Assessment (... Treating Tobacco Use and Depende...

Figure 1. HCSIT Main Screen (A); HCSIT Main Screen (scroll down) (B)

of the Public Health Service guidelines. The postsurvey also included a 4-item software usability questionnaire. Additional data on usability were obtained by conducting a group semistructured interview after completion of the postsurveys. During this session, participants were asked if there was any additional software content they would like to see added and they were asked if they had any difficulties with the software or hardware.

\section{Test}

Question 2 of 6:

Do you find it difficult to refriain from smoking in places where it is

forbidden (eg. in church, at the library, in a cinemo)?

\section{Select Answer Below:}

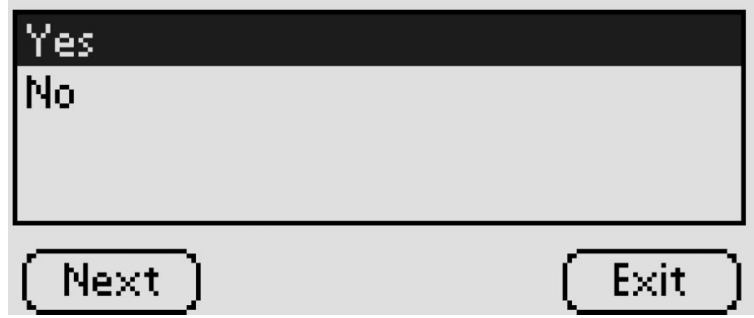

Figure 2. Sample Fagerström Nicotine Dependence Question

Study participants included 23 faculty and resident physicians from the Department of Family Medicine at the University of Virginia (UVA) Health System. These participants were selected from an initial pool of 33 faculty and resident physicians within the UVA Department of Family Medicine through a handheld usage survey. Inclusion criteria for clinicians to participate in the study were self-reports of using their handheld computers at least several times per week for at least one of

\section{Recults}

\section{Your total is: 7 .}

Scores of more than 6 generally are interpreted as indicating a high degree of dependence, with more severe withdrawal symptoms. greater difficulty in quitting. and possibly the need for higher dose of medication. As always, use good clinical judgement in your decision making. and do not prescribe any

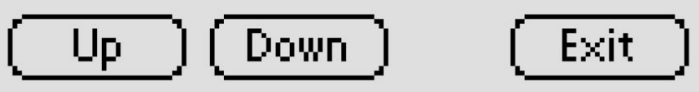

Figure 3. Fagerström Dependency 


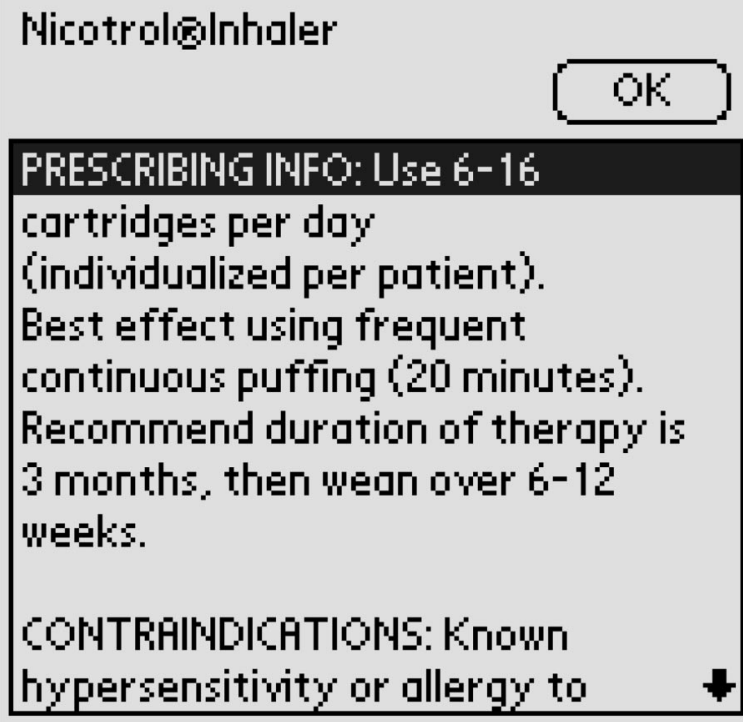

Figure 4. Drug Prescribing Information

the following functions: documentation of procedures performed, checking medication dosage, checking medication interactions, checking medical references (eg, 5 Minute Clinical Consult, Merck Manual, others), medical calculations (eg, $\mathrm{ABG}$ ), patient billing, scheduling/appointments (personal), scheduling/appointments (patients), memo pad, to-do list, addresses/phone numbers, ob calculations/tracking, recording inpatient/outpatient data, or any "other" functions that were selfreported.

The study was conducted with patients in the Department of Family Medicine's Primary Care Center and 2 affiliated rural clinics. The center is staffed by 11 faculty physicians and is the main practice site for residents. Annual patient volume is approximately 25,800 and includes patients of all ages, with a diverse ethnic makeup that includes $51 \%$ minorities. Payor mix includes 44\% Medicare and Medicaid. One affiliate practice, Stoney Creek, is located in a Health Professions Shortage Area and has a patient volume of approximately 13,400 patients per year including patients of all ages, with minorities comprising 15\%. Payor mix includes $32 \%$ Medicare and Medicaid at this site. The second affiliate practice, Crossroads Family Practice, has a patient volume of approximately 5600 patients per year including patients of all ages with minorities comprising $27 \%$. The payor mix at this site includes 33\% Medicare and Medicaid.
The 23 eligible participants were administered a presurvey. After a 30-minute education session about how to use the software, the HCSIT was provided to participants for a 4-month trial period, after which the postsurvey was administered. The education session included information on how to use the handheld program and highlighted the various topics and information available. In addition, physicians were asked to use the program in their regular clinics to assist with smoking cessation counseling.

Paired samples $t$ tests were used to assess mean differences in the 4 main subscales (physician behavior, attitudes, comfort related to counseling patients about smoking cessation, and knowledge) from pretest to posttest. An $\alpha$ level of 0.05 was used to determine statistical significance for each of the $t$ tests and a Bonferroni correction procedure was applied to account for the inflation in type 1 error due to multiple significance tests. All analyses were run using SPSS for Windows version 11.

\section{Results}

A total of 22 of 23 participants completed the pre/post survey, for a response rate of $96 \%$. Of the pre/post responders, 15 were faculty and 8 were residents. The one nonresponse was due to the person moving from the area. Participants' ages ranged from 27 to 54 . Seventy-four percent were male, and $65 \%$ reported their handheld computer literacy as intermediate on a 3-point scale from novice to expert. Reliability of the HCSIT survey subscales were the following: physician behavior $=$ 0.74 ; physician attitude $=0.52$; physician comfort $=0.71$; usability of software $=0.85$; knowledge $=0.23$. Cronbach's $\alpha$ was used for the first 4 subscales. The KR-20 was used for the knowledge subscale because the items were dichotomous. Of note is the fact that the reliability estimate for the knowledge subscale was negative. The occurrence of a negative reliability coefficient raises the possibility that sampling error was responsible for a negative average covariance among the scale items for this sample of cases. The limited number of items comprising the subscale and the small sample size also lend support for these findings.

No statistically significant mean differences were found for physician behavior (mean increase $=0.44, P=.55$ ), physician attitude (mean increase $=0.44, P=.16$ ), or physician knowledge 
1. How would you describe your hand-held computer literacy?
a. Novice
b. Intermediate
c. Expert

2. What percentage of your patients smoke? (Please check one)
$\square<10 \%$
$\square 10 \%$
$\square 20 \%$
$\square 30 \%$
$\square 50 \%$
$60 \%$
$70 \%$
$80 \%$
$40 \%$
$90 \%$
$>90 \%$

3. How often do you:

a. Ask patients if they smoke?

$\square$ Never

b. Advise smokers to quit?

$$
\square \text { Never }
$$

c. Assess smokers' willingness to quit?

$$
\square \text { Never }
$$

Sometimes

Often

Always

d. Assist smokers in their attempt to quit?

$\square$ Never
e. Arrange follow-up with patients who want to quit?

Sometimes

Often

Always

4. How often do you:
$\square$ Never
$\square$ Sometimes

a. Provide counseling for patients who want to quit smoking?
$\square$ Never
$\square$ Sometimes
Often
Always

b. Have sufficient time available with smoking patients to counsel them on initiating and sustaining a smoking cessation plan?

$\square$ Never $\quad \square$ Sometimes $\square$ Often $\square$ Always

c. Provide information on quitting such as pamphlets, videos, or online resources
$\square$ Never
Sometimes
$\square$ Often
$\square$ Always

d. Recommend pharmacotherapy for your patients who want to quit?

$\square$ Never $\quad \square$ Sometimes $\square$ Often $\square$ Always

5. If you recommend pharmacotherapy, what do you recommend? (Check all that apply)

$\square$ Nicotine Patch $\square$ Patch $\quad \square$ Inhaler $\square$ Nasal spray
$\square$ Bupropion (Wellbutrin/Zyban)

6. I have a specific intervention plan(s) I use with patients who want to quit smoking.
$\square$ Yes
$\square$ No

7. Are you familiar with the Public Health Service Clinical Practice Guidelines on smoking cessation?

$$
\square \text { Yes } \quad \square \text { No }
$$

8. The importance I place on identifying patients who smoke is

$\begin{array}{lllllll}1 & 2 & 3 & 4 & 5 & 6 & 7\end{array}$

Very unimportant

9. The importance I place on counseling patients who smoke is

$\begin{array}{lllllll}1 & 2 & 3 & 4 & 5 & 6 & 7\end{array}$

Very unimportant

10. How comfortable are you counseling patients about smoking cessation?

$\begin{array}{lllllll}1 & 2 & 3 & 4 & 5 & 6 & 7\end{array}$

Very uncomfortable $\quad$ Very comfortable

11. How comfortable are you with the Public Health Service Clinical Practice Guidelines?

$\begin{array}{lllllll}1 & 2 & 3 & 4 & 5 & 6 & 7 \\ \text { Very uncomfortable } & & & & \text { Very comfortable }\end{array}$

12. What is your comfort level with the pharmacological therapies available to aid smokers with quitting smoking?

$\begin{array}{lllllll}1 & 2 & 3 & 4 & 5 & 6 & 7 \\ \text { Very uncomfortable } & & & & \text { Very comfortable }\end{array}$

13. How comfortable are you following patients who have initiated a smoking cessation plan to help them sustain their plan?

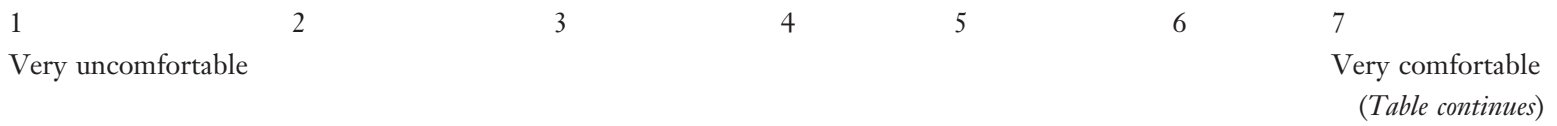




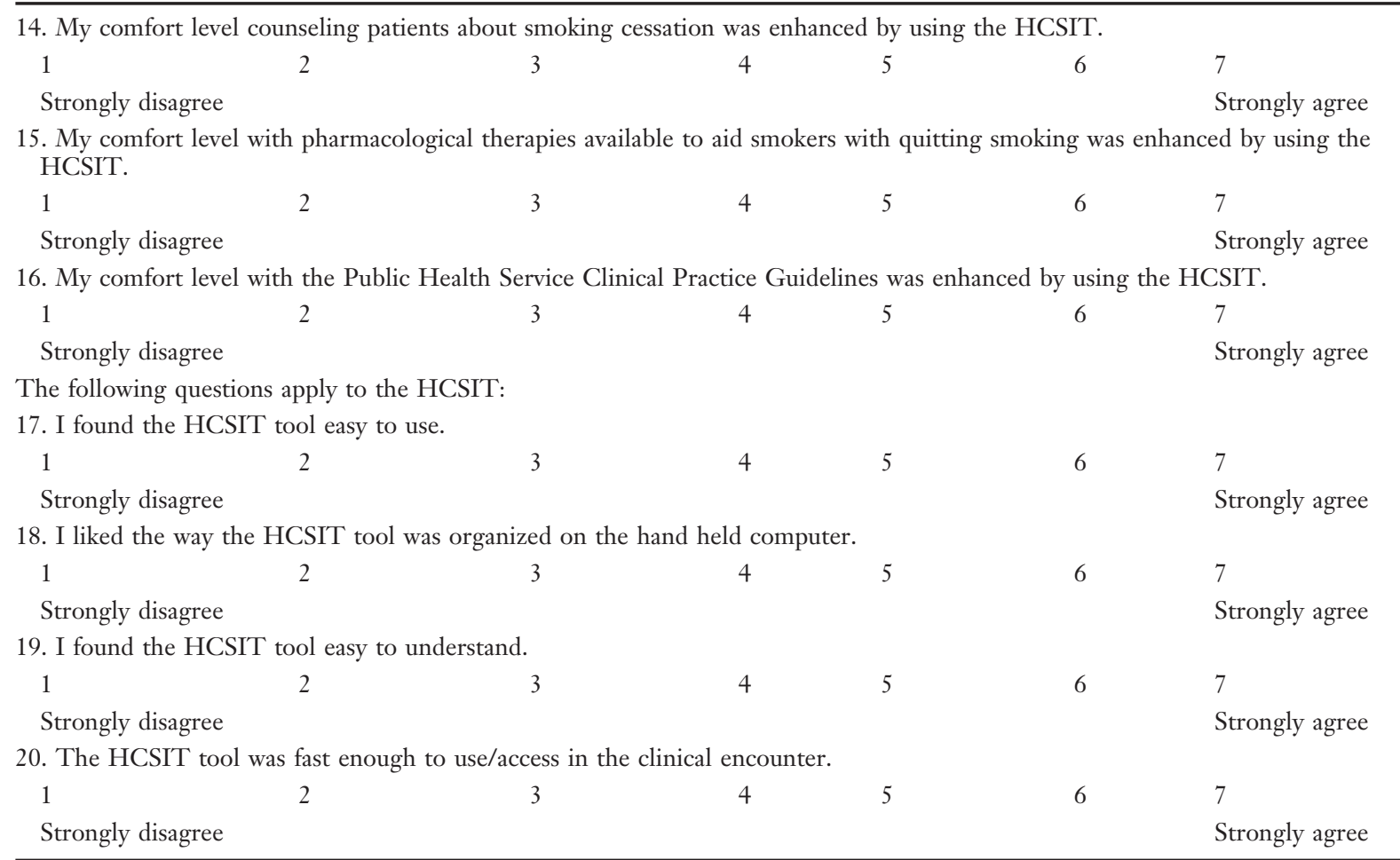

HSCIT, hand-held smoking cessation intervention tool.

(mean increase $=0.07, P=.584$ ) from pretest to posttest. However, a statistically significant mean increase of 2.29 was observed for the physician comfort subscale from pretest to posttest $(t=3.87$, $d f=16, P=.001)$. The comfort subscale was composed of 4 items as shown in Table 2. Physicians indicated improved comfort in counseling patients about smoking cessation $(P=.007)$ and improved comfort in using the Public Health Service Clinical Practice Guidelines $(P=.012)$.

Twenty of the 22 respondents (91\%) reported using the software. Actual user data were obtained for 14 of 22 physicians. Other user data were not available because of hardware and software failures. The program was used 150 times by these 14 providers, representing an average of 10.7 uses per physician. The most commonly used sections of the program were the smoking dependency assessment (14 uses), Commit prescribing information (12 uses), motivational smoking interventions (11 uses), and the treatment guidelines overview (11 uses).

Physicians found the HCSIT tool easy to use $($ mean $=4.85)$, well organized $($ mean $=4.95)$, easy to understand (mean $=5.25$ ), and fast enough for use in a clinical encounter (mean $=4.45)$. These questions were scored using a 7-point numeric scale $(1=$ strongly disagree; $7=$ strongly agree $)$. From the semistructured interview, additional content that was requested included: pregnancy and lactation information for smoking cessation pharmacotherapy; identification of the various drugs (eg, patch, inhaler, etc); and more scripting of the

Table 2. Comfort Subscale*

\begin{tabular}{lccr}
\hline Item & Mean Pretest Score & Mean Posttest Score & $P$ Value \\
\hline Counseling patients & 5.32 & 5.95 & $P=.007$ \\
Public Health Service guidelines & 3.2 & 4.35 & $P=.012$ \\
Smoking cessation drugs & 5.73 & 5.64 & $P=.605$ \\
Following patients who quit & 5.14 & 5.55 & $P=.119$ \\
\hline
\end{tabular}

* Comfort was assessed using a 7-point Likert scale with 1 representing 'very uncomfortable' and 7 representing 'very comfortable.' 
interventions was suggested. There were no reported hardware or software issues related to the HCSIT program.

\section{Discussion}

Excellent evidence-based guidelines for smoking cessation counseling by physicians have been available for nearly 2 decades. The most recent guidelines were published in the year 2000 and included a systematic review of nearly 6000 articles. ${ }^{13}$ Yet, despite this availability of clinical evidence, agencies such as the National Cancer Institute (NCI) and the Agency for Health Research and Quality (AHRQ) have struggled with translating guidelines into clinical care. In the 1980s, the NCI spent nearly $\$ 10$ million on distributing smoking cessation guidelines with little effect on physician practices. ${ }^{25}$ Similar approaches by AHRQ have met with limited success. This is consistent with other published studies that examine the lag time between the availability of clinical evidence and its actual utilization in practice, with estimates ranging from a minimum of 1 to 2 years to 13 years. ${ }^{26,27}$ The approach described in this study begins to address this difficult issue of translating smoking cessation guidelines into clinical practice by providing the relevant information to physicians at the point of care in a format that is portable, easily accessible, and convenient.

Our approach is the first known study that measures changes in physician behavior, attitude, comfort level toward smoking cessation counseling, and knowledge as a result of using a handheld computer software program at the point of care. Results indicated that physician comfort level in counseling patients about smoking cessation could indeed be improved through the utilization of specialized computer software, even among experienced clinicians. When used in conjunction with other widely available practice modifications, this tool has the potential to improve physician smoking intervention practices. Complementary practice modifications could include: using smoking as a vital sign in the patient's clinical record; Internet-based, patient-tailored self-help materials such as the American Academy of Family Physician's patient smoking cessation guides ( $\langle$ www.aafp.org $\rangle)$ or the NCI's "Clearing the Air: Quit Smoking Today" (〈www. smokefree.gov $\rangle$ ); and toll-free quit lines (such as the NCI's 1-800-Quitnow). The methodology of de- livering medical information through handheld computer technology also holds promise because of the widespread use of handheld computers by physicians; because up to $50 \%$ of physicians currently use handheld computers. ${ }^{18}$

There are some limitations to this study. The reliance on a pre/post design cannot account for other practice changes and possible confounding variables. In addition, this design was dependent on physician self-report, thereby making it susceptible to over-reporting because of social desirability and recall bias. Finally, the sample size is small and limited to family physicians that have high selfreported handheld computer usage and moderate to high computer skills, thereby limiting generalizability. This group was purposefully chosen to obtain usability data and to further refine the software program for future interventional studies among medical students and with less experienced clinicians. The small sample size also limits our ability to determine whether the tool had an effect on actual physician behavior, thus raising the possibility of a type II error. However, we were encouraged that there was a trend toward increasing the desired behaviors and improving the attitudes among these physicians toward smoking cessation counseling.

\section{Conclusion}

The use of guidelines and other medical information adapted for handheld computers holds promise in affecting physician behavior at the point of care. This study demonstrated improved physician comfort levels in providing smoking cessation counseling as a result of using a smoking intervention tool designed specifically for handheld computers. We would recommend further evaluation of this software in larger samples, including other primary care specialties (eg, general internal medicine) and other health care providers to determine generalizability, along with measuring patient outcomes. In addition, more research is needed to determine what combination and type of continuing medical education, resources, and tools will be the most helpful in improving physician smoking intervention practices.

\section{References}

1. U.S. Department of Human Services. Reducing the health consequences of smoking: 25 years of progress: A report of the surgeon general. Rockville 
(MD): U.S. Department of Health and Human Services. Public Health Services, Centers for Disease Control, Center for Chronic Disease Prevention and Health Promotion, Office on Smoking and Health; 1989; Publication (CDC) 89-8411.

2. Centers for Disease Control. Physician and other health-care professional counseling of smokers to quit-United States 1991. MMWR Morb Mortal Wkly Rep 2004;42:854-7.

3. Rigotti NA. Treatment of tobacco use and dependence. N Engl J Med 2002;346:506-12.

4. Lancaster T, Stead L, Silagy C, Sowden A. Effectiveness of interventions to help people stop smoking: Findings from the Cochrane library. BMJ 2000; 321:355-8.

5. Thorndike AN, Rigotti NA, Stafford RS, Singer DE. National patterns in the treatment of smokers by physicians. JAMA 1998;279:604-8.

6. Lewis CE, Clancy C, Leake B, Schwartz JS. The counseling practices of internists. Ann Intern Med 1991;114:54-8.

7. Goldstein MG, DePue JD, Monroe AD, et al. A population-based survey of physician smoking cessation counseling practices. Prev Med 1998;27:720-9.

8. Jaen CR, Stange KC, Nutting PA. Competing demands of primary care: a model for the delivery of clinical preventive services. J Fam Pract 1994;38: $166-71$.

9. Jaen CR, Stange KC, Tumiel LM, Nutting P. Missed opportunities for prevention: smoking cessation counseling and the competing demands of practice. J Fam Pract 1997;45:348-54.

10. Goldstein B, Fischer PM, Richards JW, Goldstein A, Shank JC. Smoking practices of recently trained family physicians. J Fam Pract 1987;24:195-7.

11. Ockene JK. Physician-delivered interventions for smoking cessation: strategies for increasing effectiveness. Prev Med 1987;16:723-37.

12. Orlandi MA. Promoting health and preventing disease in health care settings: an analysis of barriers. Prev Med 1987;16:119-30.

13. Fiore MC, Bailey WC, Cohen SJ, et al. Treating tobacco use and dependence. Clinical practice guideline. Rockville (MD): U.S. Department of Health and Human Services; 2000.

14. Heatherton TF, Kozlowski LT, Frecker RC, Fagerstrom KO. The Fagerstrom test for nicotine dependence: a revision of the Fagerstrom tolerance questionnaire. Br J Addict 1991;86:1119-27.
15. Pomerleau CS, Carton SM, Lutzke ML, et al. Reliability of the Fagerstrom tolerance questionnaire and the Fagerstrom test for nicotine dependence. Addict Behav 1994;19:33-9.

16. Prokhorov AV, De Moor C, Pallonen UE, Hudmon KS, Koehly L, Hu S. Validation of the modified Fagerstrom tolerance questionnaire with salivary cotinine among adolescents. Addict Behav 2000;25: 429-33.

17. Baumgart DC. Personal digital assistants in health care: experienced clinicians in the palm of your hand?. Lancet 2005;366:1210-22.

18. Bishop LS, Brown EG. Usability holds back MD handheld usage. Forrester Research; 2005. Available from: http://www.forrester.com/Research/Document/Excerpt/0,7211,36530,00.html. Accessed January 24, 2006.

19. Sintchenko V, Iredell JR, Gilbert GL, Coiera E. Handheld computer-based decision support reduces patient length of stay and antibiotic prescribing in critical care. J Am Med Inform Assoc 2005;12:398402.

20. Shiffman RN, Freudigman M, Brandt CA, Liaw Y, Navedo DD. A guideline implementation system using handheld computers for office management of asthma: effects on adherence and patient outcomes. Pediatrics 2000;105:767-73.

21. Rothschild JM, Lee TH, Bae T, Bates DW. Clinician use of a palmtop drug reference guide. J Am Med Inform Assoc 2002;9:223-9.

22. Christakis DA, Zimmerman FJ, Wright JA, Garrison MM, Rivara FP, Davis RL. A randomized controlled trial of point-of-care evidence to improve the antibiotic prescribing practices for otitis media in children. Pediatrics 2001;107:E15.

23. Samore MH, Bateman K, Alder SC, et al. Clinical decision support and appropriateness of antimicrobial prescribing: a randomized trial. JAMA 2005;294: 2305-14.

24. Green LA. Prescription for health: round 1 initial results. Ann Fam Med 2005;3 Suppl 2:S1-68.

25. Mohon MA. Do physicians counsel smokers to quit? J Okla State Med Assoc 2000;93:103-6.

26. Fineberg HV. Clinical evaluation: how does it influence medical practice? Bull Cancer 1987;74:333-46.

27. Antman EM, Lau J, Kupelnick B, Mosteller F, Chalmers TC. A comparison of results of metaanalyses of randomized control trials and recommendations of clinical experts treatments for myocardial infarction. JAMA 1992;268:240-8. 